\title{
QUALIDADE DO SUBSTRATO MINERADO DE UMA ÁREA DE EMPRÉSTIMO REVEGETADA NO DISTRITO FEDERAL $^{(1)}$
}

\author{
Rodrigo Studart Corrêa ${ }^{(2)} \&$ Marcel Anderson Borges Bento ${ }^{(3)}$
}

\begin{abstract}
RESUMO
O uso de resíduos orgânicos tem-se mostrado eficaz para a revegetação de áreas mineradas. Entretanto, dados acerca da qualidade de substratos tratados com resíduos orgânicos são escassos. Dessa forma, este trabalho visou avaliar a qualidade edáfica de um substrato minerado no Distrito Federal que recebeu $250 \mathrm{Mg} \mathrm{ha}^{-1}$ de lodo de esgoto e uma cobertura herbácea estabelecida a partir de sementes. Os atributos edáficos utilizados na avaliação foram densidade de solo, porosidade total, água disponível, resistência mecânica à penetração, $\Delta$ pH, CTC, saturação por bases, $P$ disponível, matéria orgânica, abundância e diversidade da macrofauna de solo. Os resultados mostraram que a incorporação do lodo de esgoto e o desenvolvimento de um estrato herbáceo resultaram em melhoria das qualidades química e biológica do substrato exposto pela mineração, mas não alteraram a qualidade física dele. A dose de lodo de esgoto utilizada elevou os valores dos atributos químicos para além dos medidos em solos sob Cerrado nativo. Caso o objetivo da recuperação seja a restauração do ecossistema local, técnicas que melhorem a qualidade física do substrato e não excedam os níveis naturais de fertilidade devem ser desenvolvidas.
\end{abstract}

Termos de indexação: revegetação, mineração, qualidade do solo, biossólidos.

SUMMARY: QUALITY OF A REVEGETED MINE SPOIL IN THE FEDERAL DISRICT OF BRAZIL

The use of organic residues has been shown to be effective for the revegetation of mined areas. However, data on the quality of mine spoils treated with organic residues are rather scarce. This study aimed to evaluate the spoil quality of a mined area in the Brazilian Federal

\footnotetext{
(1) Parte da Dissertação de Mestrado do segundo autor. Recebido para publicação em setembro de 2009 e aprovado em junho de 2010.

(2) Pesquisador do Departamento de Ecologia e da Rede Brasileira de Recuperação Ambiental - Rebram, Universidade de Brasília - UnB. Caixa Postal 04401, CEP 70910-900 Brasília (DF). Bolsista de Produtividade em Pesquisa do CNPq. E-mail: rodmanga@yahoo.com.br

(3) Mestre em Ciências Florestais e Engenheiro Florestal, UnB. E-mail: marcelbentoxvi@gmail.com
} 


\begin{abstract}
District treated with $250 \mathrm{Mg} \mathrm{ha}^{-1}$ of domestic sewage sludge and covered with herbs grown from seeds. The soil attributes used for the evaluation were bulk density, total porosity, available water capacity, soil resistance to penetration, $\triangle p H, C E C$, base saturation, available phosphorus, organic matter content, abundance and diversity of soil macro-fauna. Results show that plowing sewage sludge into the spoil and the establishment of a herbaceous layer improved the chemical and biological qualities but not the physical one. The rate of sewage sludge applied to the spoil increased the values of the chemical parameters to more than normally measured for soils under native Cerrado vegetation. If revegetation is focused on the restoration of the local ecosystem, techniques to improve spoil physical quality and without exceeding natural fertility levels must be developed.
\end{abstract}

Index terms: revegetation, mining, soil quality, biosolids.

\section{INTRODUÇÃO}

A urbanização do Distrito Federal - DF resultou em $0,6 \%$ de sua extensão degradada pela mineração (Corrêa et al., 2004). A exploração de materiais para a construção civil (areia, brita, argila, cascalho e outros) expôs à superfície material de composição variada, genericamente denominado substrato. Substratos expostos pela mineração apresentam atributos físicos, químicos e biológicos diferentes do solo, que possui camadas em seu perfil e características adequadas para o crescimento e desenvolvimento de plantas e outros organismos (Goedert \& Corrêa, 2004).

A implantação de uma cobertura vegetal sobre superfícies mineradas é a medida mais comum de recuperação, mas requer a construção de um ambiente edáfico que seja compatível com as espécies vegetais a serem utilizadas. Reabilitar ecossistemas é a denominação atribuída ao desafio de se proverem condições topográficas, edáficas e biológicas para o restabelecimento de processos ecológicos em um local degradado (Alves et al., 2007). Escarificação, subsolagem, gradagem, incorporação de matéria orgânica e de insumos químicos são ações que visam à reabilitação de substratos minerados (Reis, 2006; Calgaro et al., 2008), e a qualidade do novo ambiente edáfico dependerá das características do material exposto à superfície e dos processos utilizados na sua recuperação (Campos et al., 2003).

As técnicas utilizadas para a recuperação de áreas escavadas são variadas (Valcarcel et al., 2007) e a incorporação de fontes de matéria orgânica a substratos é uma delas (Leite et al., 1994). A matéria orgânica melhora as características físicas do material exposto, eleva a sua fertilidade e serve de meio para o estabelecimento e manutenção de microrganismos e fauna de solo (Vargas \& Hungria, 1997; Pignataro Netto et al., 2009). Por esses motivos, o lodo de esgoto produzido no Distrito Federal tem sido utilizado desde 1994 como fonte de matéria orgânica em trabalhos de revegetação de jazidas explotadas. Esse resíduo é um subproduto do tratamento de esgotos domésticos de Brasília e apresenta grande potencial fertilizante e condicionador de solos (Adani et al., 2007). Além disso, em regiões tropicais, a maior parte da capacidade de troca de cátions - CTC dos solos (70-80\%) depende da matéria orgânica (Brady \& Weil, 2002), pois ela altera o complexo coloidal, criando cargas (Bezerra et al., 2006). Incrementos de CTC, para valores de até $16 \mathrm{cmol}_{\mathrm{c}} \mathrm{dm}^{-3}$, e das concentrações de nutrientes são efeitos relatados em solos e substratos tratados com lodo de esgoto (Corrêa et al., 2010).

O uso de resíduos orgânicos para o estabelecimento de uma cobertura vegetal sobre superfícies expostas pela mineração tem-se mostrado eficaz em diferentes ecossistemas (Araujo, 2006; Bezerra et al., 2006; Adani et al., 2007; Corrêa et al., 2010). Todavia, pesquisas acerca da qualidade dos substratos revegetados com o uso desses materiais são escassas, apesar de necessárias para um melhor manejo de resíduos orgânicos em áreas mineradas. Qualidade edáfica pode ser entendida como a capacidade de um solo exercer suas funções na natureza, e ela pode ser avaliada por meio da mensuração de atributos físicos, químicos e biológicos (Doran \& Zeiss, 2000). Dessa forma, este trabalho objetivou avaliar a qualidade edáfica de um substrato minerado no Distrito Federal, que fora revegetado há mais de uma década com o uso de lodo de esgoto como fonte de matéria orgânica e de nutrientes.

\section{MATERIAL E MÉTODOS}

\section{Localização e histórico da área}

O estudo foi realizado em uma caixa de empréstimo explorada em 1955, de onde se retirou material argiloso para a construção da barragem que forma o Lago Paranoá, em Brasília. A jazida de 15 ha situa-se nas coordenadas de $15^{\circ} 48^{\prime} 44^{\prime} \mathrm{S}$ e $47^{\circ} 47^{\prime} 34^{\prime}$ 'W e apresentava antes da mineração Latossolo Vermelho como solo original (Embrapa, 1999). O clima predominante na área é o Tropical de Savana - Aw, segundo a classificação de Köppen. A precipitação pluvial anual varia de 1.200 a $1.600 \mathrm{~mm}$, com $84 \%$ do volume sendo precipitado no verão. A temperatura média anual oscila entre 18 e $22^{\circ} \mathrm{C}$, e a umidade relativa do ar varia de 12 a $85 \%$. 
O local de estudo permaneceu desprovido de cobertura vegetal até 1997, quando $250 \mathrm{Mg} \mathrm{ha}^{-1}$ de lodo de esgoto da Estação de Tratamento de Esgoto Brasília Sul, operada pela Companhia de Saneamento Ambiental do Distrito Federal - Caesb, foram incorporados ao substrato. Tratava-se de uma mistura de lodo originado pelos tratamentos primário, secundário (lodo biologicamente ativado) e terciário [polido com $\mathrm{Al}_{2}\left(\mathrm{SO}_{4}\right)_{3}$ ] (Quadro 1). O resíduo foi incorporado na camada de $0-20 \mathrm{~cm}$ de profundidade com uma grade pesada. Cal hidratada $(\mathrm{CaOH})$ foi aplicada à superfície da área $\left(20 \mathrm{Mg} \mathrm{ha}^{-1}\right)$ para controle sanitário de vetores. Um mistura composta por sementes de Cajanus cajan, Crotalaria spp., Mucuna aterrima e Stizolobium sp. foi incorporada ao substrato tratado, em dose de $100 \mathrm{~kg} \mathrm{ha}^{-1}$. Nos anos que se sucederam (1998-2008), a superfície da área foi coberta por espécies herbáceas diversas que naturalmente se estabeleceram no local. O capim Brachiaria decumbens respondia na época da coleta de dados por quase a totalidade da cobertura vegetal da jazida.

\section{Avaliação da qualidade do substrato}

As condições física, química e biológica do substrato avaliadas em 2008 são resultados da incorporação mecânica de lodo de esgoto e do estabelecimento de uma cobertura vegetal herbácea em 1997. A qualidade do substrato revegetado foi avaliada por meio da mensuração de atributos físicos, químicos e biológicos (Doran \& Zeiss, 2000), que também foram mensurados em um Latossolo Vermelho (Embrapa, 1999) sob Cerrado, em local adjacente, para efeito de controle.

\section{Qualidade física}

A qualidade física do substrato revegetado foi determinada por meio de propriedades utilizadas para descrever solos (Alves et al., 2007): densidade, porosidade, resistência à penetração e água disponível. Amostras indeformadas do substrato revegetado e de solo sob Cerrado nativo foram coletadas entre 0 e $20 \mathrm{~cm}$ de profundidade, para cálculo da densidade de solo $\left(\rho_{\mathrm{b}}\right)$ pelo Método do Anel Volumétrico (Base Úmida) $-100 \mathrm{~cm}^{3}$ (Kopecky). A porosidade total (Pt) foi

Quadro 1. Principais características do lodo de esgoto (base úmida) incorporado ao substrato exposto da caixa de empréstimo (média \pm desviopadrão)

\begin{tabular}{lc}
\hline Característica & Lodo de esgoto \\
\hline Umidade $\left(\mathrm{dag} \mathrm{kg}{ }^{-1}\right)$ & $87,5 \pm 4,3$ \\
Matéria orgânica $\left(\mathrm{g} \mathrm{kg}^{-1}\right)$ & $439 \pm 2,1$ \\
Nitrogênio total $\left(\mathrm{g} \mathrm{kg}^{-1}\right.$ & $53,5 \pm 2,7$ \\
Fósforo total $\left(\mathrm{g} \mathrm{kg}^{-1}\right)$ & $17,5 \pm 0,8$ \\
Potássio total $\left(\mathrm{g} \mathrm{kg}^{-1}\right)$ & $1,8 \pm<0,01$ \\
Cálcio total $\left(\mathrm{g} \mathrm{kg}^{-1}\right)$ & $26,8 \pm 1,4$ \\
Magnésio total $\left(\mathrm{g} \mathrm{kg}^{-1}\right)$ & $4,1 \pm 0,3$ \\
Enxofre total $\left(\mathrm{g} \mathrm{kg}^{-1}\right)$ & $6,2 \pm 0,9$ \\
\hline
\end{tabular}

calculada por meio da equação 1 , que estabelece a relação entre a densidade de solo $\left(\rho_{\mathrm{b}}\right)$ e a densidade de partículas de solo, considerando-se o valor fixo de 2,65 $\mathrm{Mg} \mathrm{m}^{-3}$, conforme Embrapa (1997):

$$
\operatorname{Pt}\left(\mathrm{m}^{3} \mathrm{~m}^{-3}\right)=1-\frac{\rho_{\mathrm{b}}}{2,65}
$$

A capacidade de campo (CC) e o ponto de murcha permanente (PMP) do substrato e do solo sob Cerrado foram mensurados na camada de 0-20 cm, em mesa de tensão, a $10 \mathrm{kPa}(\mathrm{CC})$ e $1.500 \mathrm{kPa}(\mathrm{PMP})$, com a utilização de cilindros metálicos de $100 \mathrm{~cm}^{3}$ (Embrapa, 1997). Os volumes de água disponível (AD) do substrato e do solo foram calculados pela diferença entre o volume de água armazenado à $\mathrm{CC}$ e o armazenado ao PMP. A resistência à penetração $(\mathrm{Rp})$ foi avaliada por meio de um penetrômetro de impacto, modelo IAA/PLANALSUCAR (Stolf et al., 1983). Os testes de $\mathrm{Rp}$ foram realizados no período chuvoso de 2008 , entre 0 e $30 \mathrm{~cm}$ de profundidade, em cinco repetições. Os resultados obtidos no campo foram convertidos conforme a equação 2 (Stolf, 1991):

$$
\operatorname{Rp}\left(\mathrm{kgf} \mathrm{cm}^{-2}\right)=5,6+6,98 \mathrm{~N}\left(\text { impactos } \mathrm{dm}^{-1}\right)
$$

Para melhor representação dos dados, converteramse os valores de Rp obtidos com a equação 2 e expressos em kgf $\mathrm{cm}^{-2}$ para $\mathrm{MPa}$, multiplicando-se por 0,098, conforme Oliveira et al. (2007). Curvas de resistência mecânica à penetração foram traçadas ao longo da camada de $0-30 \mathrm{~cm}$, utilizando os valores médios encontrados a cada $2 \mathrm{~cm}$ de profundidade. Os teores de areia, silte e argila foram mensurados pelo método de Bouyoucos (Embrapa, 1997).

\section{Qualidade química}

A qualidade química do substrato revegetado e do solo sob Cerrado foi avaliada por meio da análise do pH em água e em $\mathrm{KCl}$, das concentrações de $\mathrm{P}$ disponível, bases $\left(\mathrm{Ca}^{2+}, \mathrm{Mg}^{2+}, \mathrm{K}^{+}, \mathrm{Na}^{+}\right)$, acidez potencial $(\mathrm{Al}+\mathrm{H}), \Delta \mathrm{pH}$, capacidade de troca de cátions (CTC a pH 7), saturação por bases (V \%) e teor de matéria orgânica, conforme métodos descritos em Embrapa (1997). Três amostras, compostas por 10 subamostras de substrato e de solo, foram coletadas entre 0 e $20 \mathrm{~cm}$ de profundidade, ensacadas, secas à sombra por duas semanas, passadas em peneira de 10 Mesh e levadas para o laboratório.

No laboratório, o $\mathrm{pH}$ das amostras foi medido com potenciômetro, em uma solução 1:2,5 de solo-água. Fósforo disponível e K trocável foram extraídos com solução Mehlich-1, e as concentrações foram determinadas em fotocolorímetro e em espectrofotômetro de absorção atômica, respectivamente. Cálcio e Mg trocáveis foram extraídos com uma solução de $\mathrm{KCl}$ e analisados por espectrofotometria de absorção atômica. A acidez potencial $(\mathrm{H}+\mathrm{Al})$ foi determinada por meio de uma solução tamponada de acetado de cálcio e posterior titulação com $\mathrm{NaOH}$. A análise de matéria 
orgânica seguiu o método da combustão úmida (Walkley-Black) e posterior titulação com uma solução de sulfato ferroso amoniacal (Embrapa, 1997). A partir dos resultados obtidos, foram calculados o $\Delta \mathrm{pH}$, a capacidade de troca de cátions (CTC a pH 7) e a percentagem de saturação por bases $(\mathrm{V} \%)$ do substrato e do solo (Embrapa, 1997). Os parâmetros mensurados no substrato exposto antes de sua revegetação em 1997 (Quadro 2) seguiram os mesmos materiais e métodos utilizados em 2008 para a análise do substrato revegetado e do solo sob Cerrado.

\section{Qualidade biológica}

A qualidade biológica do substrato revegetado e do solo sob Cerrado foi avaliada por meio da abundância e da diversidade da macrofauna de solo. Considerouse macrofauna de solo os indivíduos entre 4 e $80 \mathrm{~mm}$ de diâmetro (Vargas \& Hungria, 1997; Kaschuk et al., 2006). Dez amostras de 20 x 20 x 15 cm de substrato revegetado e dez amostras de solo sob Cerrado foram coletadas com uma pá de jardineiro. Os representantes da macrofauna foram manualmente extraídos das amostras com auxílio de pinças e lupas. Os indivíduos encontrados foram fixados em álcool $90 \%$, contados (abundância) e identificados por grupo taxonômico até o nível de Classe ou Ordem.

No cálculo da diversidade da macrofauna utilizouse o Índice de Comparação Sequencial - SCI, que considera o conceito de morfoespécie (Gray, 1999). O SCI é uma ferramenta que quantifica a diversidade de ecossistemas e compara a diversidade de diferentes locais. Ele é calculado por meio da razão entre o número sequencial de indivíduos da mesma Classe ou Ordem triados de uma amostra (A) e o total de indivíduos da amostra (B) (Gray, 1999), conforme equação 3:

$$
\mathrm{SCI}=\mathrm{A} / \mathrm{B}
$$

Após a determinação do SCI de cada amostra, determinou-se o valor médio de morfoespécies do substrato revegetado e do solo sob Cerrado $\left(\mathrm{SCI}_{\mathrm{M}}\right)$, por meio da razão entre o somatório dos índices $(\Sigma \mathrm{SCI})$ e a quantidade de amostras coletadas (n), conforme equação 4:

$$
\mathrm{SCI}_{\mathrm{M}}=\sum_{N=1}^{10} \mathrm{SCI} / \mathrm{n}
$$

\section{Tratamento dos dados}

Os resultados dos atributos avaliados foram submetidos a análise de variância, teste t ou teste de Tukey e correlações, utilizando-se o programa SYSTAT 12. A significância das correlações foi testada por meio do coeficiente de Pearson.

A qualidade de solos e substratos não deve ser avaliada por indicadores individuais, pois existem relações entre os diversos atributos do solo (Alves et al., 2007). Dessa forma, a avaliação da qualidade do substrato revegetado foi baseada em procedimento que conjuga variáveis analisadas em valores únicos de qualidade física, química e biológica, conforme método descrito por Araújo (2004) e usado por Araújo et al. (2007) e Pignaro Netto et al. (2009). Os valores dos atributos obtidos no solo sob Cerrado nativo foram considerados ideais e, portanto iguais a $100 \%$. Os

Quadro 2. Atributos físicos, químicos e biológicos do substrato revegetado e do solo sob Cerrado analisados

\begin{tabular}{|c|c|c|c|}
\hline Atributo & $\begin{array}{c}\text { Substrato exposto } \\
(1997)\end{array}$ & $\begin{array}{c}\text { Substrato revegetado } \\
(2008)\end{array}$ & $\begin{array}{c}\text { Solo sob Cerrado } \\
(2008)\end{array}$ \\
\hline & & Físico & \\
\hline$\rho b\left(\mathrm{Mg} \mathrm{m}^{-3}\right)$ & $1,32 \mathrm{a}$ & $1,32 \mathrm{a}$ & $0,93 \mathrm{~b}$ \\
\hline $\mathrm{Pt}\left(\mathrm{m}^{3} \mathrm{~m}^{-3}\right)$ & $0,50 \mathrm{a}$ & $0,50 \mathrm{a}$ & $0,65 \mathrm{~b}$ \\
\hline Capacidade de campo $\left(\mathrm{m}^{3} \mathrm{~m}^{-3}\right)$ & --- & $0,21 \mathrm{a}$ & $0,41 \mathrm{~b}$ \\
\hline Ponto de Murcha Permanente $\left(\mathrm{m}^{3} \mathrm{~m}^{-3}\right)$ & --- & $0,12 \mathrm{a}$ & $0,09 \mathrm{a}$ \\
\hline Água Disponível $\left(\mathrm{m}^{3} \mathrm{~m}^{-3}\right)$ & --- & $0,09 \mathrm{a}$ & $0,32 \mathrm{~b}$ \\
\hline Argila $\left(\mathrm{g} \mathrm{kg}^{-1}\right)$ & $490 \mathrm{a}$ & $480 \mathrm{a}$ & $123 \mathrm{~b}$ \\
\hline Silte $\left(\mathrm{g} \mathrm{kg}^{-1}\right)$ & $380 \mathrm{a}$ & $370 \mathrm{a}$ & $133 \mathrm{~b}$ \\
\hline \multirow[t]{2}{*}{ Areia $\left(\mathrm{g} \mathrm{kg}^{-1}\right)$} & $130 \mathrm{a}$ & $150 \mathrm{a}$ & $743 \mathrm{~b}$ \\
\hline & & Químico & \\
\hline pH em água & $5,2 \mathrm{a}$ & $5,5 \mathrm{a}$ & $5,2 \mathrm{a}$ \\
\hline$\Delta \mathrm{pH}$ & $1,1 \mathrm{a}$ & $-0,6 \mathrm{~b}$ & $-0,9 b$ \\
\hline CTC $\left(\mathrm{cmol}_{\mathrm{c}} \mathrm{dm}^{-3}\right)$ & $1,5 \mathrm{a}$ & $5,7 \mathrm{~b}$ & $4,3 \mathrm{c}$ \\
\hline Saturação por bases (V\%) & $25,2 \mathrm{a}$ & 29,3 a & $9,0 \mathrm{~b}$ \\
\hline $\mathrm{P}$ disponível $\left(\mathrm{mg} \mathrm{dm}^{-3}\right)$ & $<0,01$ a & $27,0 \mathrm{~b}$ & $2,1 \mathrm{c}$ \\
\hline \multirow[t]{2}{*}{ Matéria orgânica $\left(\mathrm{g} \mathrm{kg}^{-1}\right)$} & $6,1 \mathrm{a}$ & $29,7 \mathrm{~b}$ & $22,3 \mathrm{c}$ \\
\hline & & Biológico & \\
\hline Abundância ( $\mathrm{n}^{\circ}$ de indivíduos) & --- & $43,6 \mathrm{a}$ & $33,3 \mathrm{~b}$ \\
\hline Diversidade (SCIM) & -.- & $0,48 \mathrm{a}$ & $0,65 \mathrm{~b}$ \\
\hline
\end{tabular}
neste trabalho

Médias ( $\mathrm{n}=3)$ seguidas de mesma letra em cada linha não diferem pelo teste $\mathrm{t}$ ou teste de Tukey $(\mathrm{p}<0,05)$. 
valores dos atributos mensurados em 2008 no substrato revegetado foram relativizados com base no solo sob Cerrado, segundo as equações 5 e 6 :

$$
\mathrm{A}_{(\mathrm{F}, \mathrm{Q}, \mathrm{B})}=\sum \mathrm{Iz}
$$

em que

$$
\mathrm{Iz}=\mathrm{a}+\left[\mathrm{a}\left(\frac{\mathrm{Sc}-\mathrm{Sr}}{\mathrm{Sc}}\right) \mathrm{b}\right]
$$

sendo Iz (\%) - valor do indicador (z) para o substrato revegetado ( $\mathrm{Iz} \geq 0)$; $\mathrm{z}$ - atributos analisados: $\rho_{\mathrm{b}}, \mathrm{Pt}$, $\mathrm{AD}, \mathrm{Rp}, \Delta \mathrm{pH}, \mathrm{CTC}, \mathrm{V} \%$, P-disponível, matéria orgânica, abundância e diversidade $\left(\mathrm{SCI}_{\mathrm{M}}\right)$ da macrofauna de solo; a $\rightarrow 100 \% / \mathrm{n}^{\circ}$ de atributos; Sc valor do atributo avaliado no solo sob Cerrado; $\mathrm{Sr}$ valor do atributo avaliado no substrato revegetado; $\mathrm{e}$ $\mathrm{b} \rightarrow 1,0$ caso a redução do valor do atributo seja considerada desejável; -1,0 caso o aumento do valor do indicador seja considerado desejável.

Por fim, foi criado um diagrama com três vértices (triângulo), em que cada vértice representa o valor conjugado dos atributos (Iz) utilizados para o cálculo da qualidade física, química e biológica. $\mathrm{O}$ índice de qualidade do substrato revegetado (IQ) foi, dessa forma, representado pela razão entre as áreas geométricas dos triângulos formados pelos valores de qualidade do substrato revegetado e do solo sob Cerrado (Goedert \& Corrêa, 2004; Araujo, 2006; Araújo et al., 2007; Pignaro Netto et al., 2009), conforme equação 7:

$$
\mathrm{IQ}(\%)=\mathrm{A} / \mathrm{Ax}
$$

em que A - área geométrica do triângulo formado pelos atributos mensurados no substrato revegetado; e $\mathrm{A}_{\mathrm{x}}$ área geométrica do triângulo formado pelos atributos mensurados no solo sob Cerrado.

\section{RESULTADOS E DISCUSSÃO}

\section{Qualidade física}

Um dos efeitos da degradação da qualidade de solos é o aumento de sua densidade (Alves et al., 2007), e a incorporação de uma fonte de matéria orgânica e o estabelecimento de uma cobertura herbácea são medidas eficazes para melhorar as condições físicas de solos degradados (Pignataro Netto et al., 2009). Todavia, a incorporação de lodo de esgoto e a revegetação do substrato exposto não alteraram a sua densidade $\left(\rho_{b}\right)$, que permaneceu $42 \%$ superior ao valor medido no solo sob Cerrado (Quadro 2). Leite et al. (1994) reduziram a densidade de um substrato minerado de 1,6 para $1,4 \mathrm{Mg} \mathrm{m}^{-3}$ por meio da descompactação mecânica e posterior incorporação de matéria orgânica. A área deste trabalho não sofreu processos de escarificação ou subsolagem e, portanto, não houve descompactação mecânica da superfície exposta. O valor de $1,32 \mathrm{Mg} \mathrm{m}^{-3}$ medido no substrato revegetado não é considerado impeditivo para o crescimento radicular de plantas (Pignataro Netto et al., 2009), mas pode dificultar o estabelecimento natural de outras espécies vegetais (Goedert, 2005) e, consequentemente, retardar o processo de sucessão natural no local (Dajoz, 2005).

Densidade $\left(\rho_{\mathrm{b}}\right)$ e porosidade $(\mathrm{Pt})$ de solos são atributos fortemente relacionados (Goedert, 2005; Alves et al., 2007). O elevado valor de $\rho_{b}$ do substrato revegetado refletiu em valores inferiores de Pt e água disponível, quando comparados aos do solo sob Cerrado (Quadro 2). A $\rho_{\mathrm{b}}$ explicou parte do volume de água disponível (AD) no solo sob Cerrado $(r=0,56)$, mas não no substrato revegetado. A proporção entre macro e microporos é outra característica que influencia o volume de água disponível em solos (Brady \& Weil, 2002). O substrato revegetado apresentou $42 \%$ de sua $\operatorname{Pt}\left(0,50 \mathrm{~m}^{3} \mathrm{~m}^{-3}\right)$ na forma de microporos, enquanto no solo sob Cerrado $63 \%$ de $0,65 \mathrm{~m}^{3} \mathrm{~m}^{-3}$ eram de microporos. Como resultado, o substrato revegetado apresentou a metade da capacidade de o solo sob Cerrado armazenar água (CC) e apenas um terço da água disponível $(\mathrm{AD})$ para plantas e outros organismos (Quadro 2).

Aeração, infiltração, armazenamento de água, movimentação de nutrientes e o estabelecimento de plantas dependem da Pt que um solo possui (Pignataro Netto et al., 2009). De acordo com Kiehl (1979), a Pt de um solo ideal para o crescimento e desenvolvimento de plantas deve ser de $0,50 \mathrm{~m}^{3} \mathrm{~m}^{-3}$, sendo $33 \%$ de macroporos (aeração) e $67 \%$ de microporos para armazenamento de água. Em face do exposto e considerando $0,13 \mathrm{~m}^{3} \mathrm{~m}^{-3}$ como o mínimo de água disponível que um solo deve possuir (Goedert, 2005), o substrato revegetado não apresentaria condições de fornecer água em quantidade suficiente para culturas agrícolas.

Densidade, umidade, teor de matéria orgânica e textura são fatores determinantes da resistência mecânica que raízes irão enfrentar para se estabelecerem em solos (Oliveira et al., 2007). Entre esses fatores, a densidade $\left(\rho_{b}\right)$ foi o atributo mais significativo $(r=0,61)$ na determinação da resistência mecânica do substrato revegetado, seguido pelo seu teor de argila $(\mathrm{r}=0,57)$. O teor de matéria orgânica do substrato revegetado é $30 \%$ superior ao medido no solo sob Cerrado (Quadro 2), porém isso não foi suficiente para reduzir a resistência mecânica a níveis próximos aos do solo usado como controle. A resistência mecânica do substrato revegetado foi entre $1,2(30 \mathrm{~cm})$ e 3,0 $(14 \mathrm{~cm})$ vezes superior à apresentada pelo solo sob Cerrado (Figura 1). Valores de resistência mecânica à penetração a partir de $2,5 \mathrm{MPa}$ são limitantes para o crescimento e desenvolvimento adequado de plantas cultivadas (Oliveira et al., 2007). $\mathrm{O}$ solo sob Cerrado apresentou valores de resistência inferiores a $2,5 \mathrm{MPa}$, e o substrato revegetado, 
superiores a 2,5 $\mathrm{MPa}$, exceto na camada superficial de 0 a $4 \mathrm{~cm}$ de profundidade (Figura 1). Após uma década da incorporação de material orgânico e do estabelecimento de uma camada herbácea sobre o substrato minerado, seus atributos físicos situavamse entre 30 e $77 \%$ dos valores medidos no solo sob Cerrado, usado como referência (Quadro 2). O valor do indicador (Iz) de qualidade física para o substrato revegetado limitou-se, portanto, a $61 \%$ da qualidade mensurada no solo sob Cerrado (Figura 2).

\section{Qualidade química}

$\mathrm{O}$ pH indica as condições gerais de fertilidade do solo (Brady \& Weil, 2002), e a disponibilidade de nutrientes é fortemente influenciada por ele (Malavolta, 1987). Dos 11 atributos químicos analisados no substrato revegetado [pH em água, $\mathrm{pH}_{\mathrm{KCl}}$, P-disponível, $\mathrm{Ca}^{2+}, \mathrm{Mg}^{2+}, \mathrm{K}^{+}, \mathrm{Na}^{+},(\mathrm{H}+\mathrm{Al})$, CTC, V \% e matéria orgânica], o pH em água relacionou-se significativamente com nove deles: acidez potencial $(r=0,97), \mathrm{CTC}(r=0,91), \mathrm{Ca}^{2+}(r=0,88), \mathrm{P}$ disponível $(r=0,86)$, matéria orgânica $(r=0,74), \mathrm{Mg}^{2+}$ $(\mathrm{r}=0,69), \mathrm{pH}_{\mathrm{KCl}}(\mathrm{r}=0,68), \mathrm{V} \%(\mathrm{r}=0,63)$ e K-trocável $(r=0,52)$. Contudo, a mudança mais proeminente

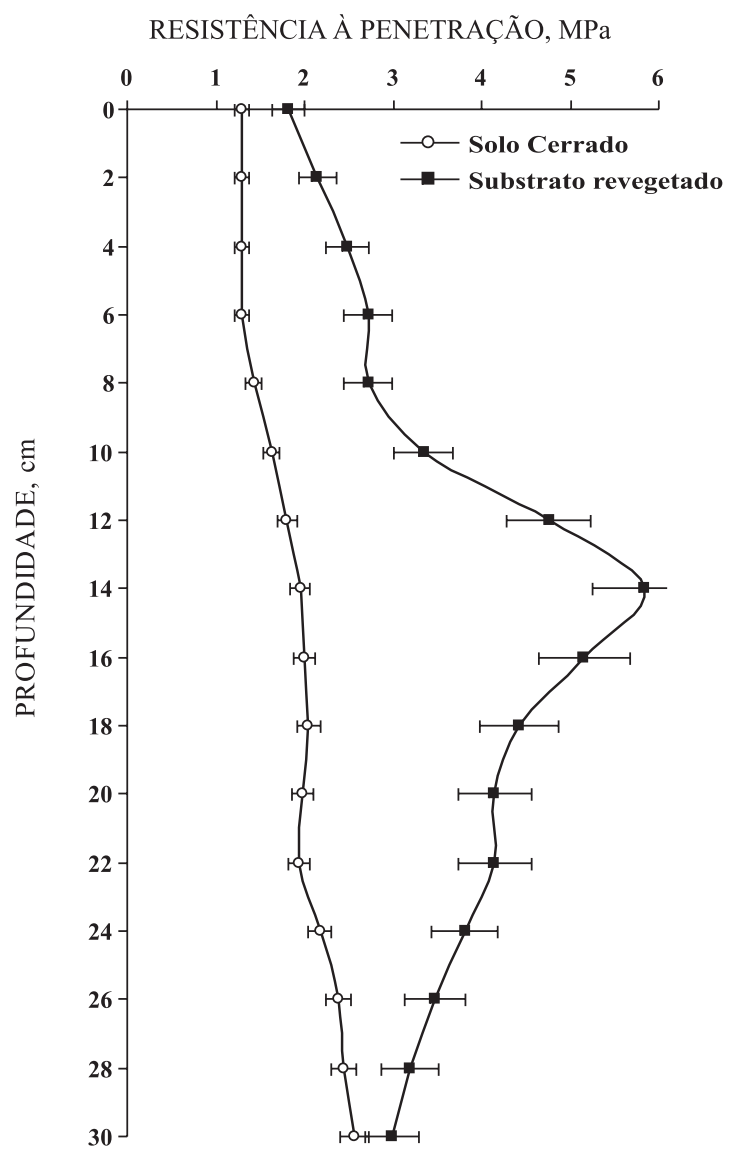

Figura 1. Resistência mecânica à penetração para o substrato revegetado e para o solo sob Cerrado nativo.

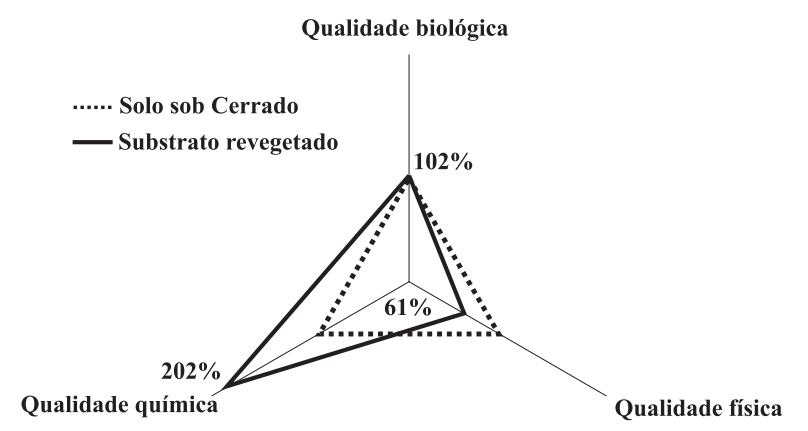

Figura 2. Diagrama comparativo da qualidade do substrato revegetado em relação ao solo sob Cerrado.

relacionada ao $\mathrm{pH}$ refere-se ao $\Delta \mathrm{pH}$, que refletia uma condição de predominância de cargas positivas em 1997, alterada para uma predominância de cargas negativas em 2008 (Quadro 2). A baixa fertilidade e as condições inapropriadas ao estabelecimento de plantas do substrato exposto (1997) podem ser confirmadas por seus valores de CTC e P-disponível (Quadro 2).

A revegetação de áreas mineradas não é possível sem que haja a reabilitação das funções edáficas no substrato exposto, e a elevação da CTC é essencial para esse processo (Goedert \& Corrêa, 2004). O aumento de CTC de solos e substratos tratados com lodo de esgoto é um efeito da matéria orgânica que esse resíduo contém (Simonete et al., 2003; Bezerra et al., 2006; Corrêa et al., 2010). A matéria orgânica do lodo de esgoto contribuiu para quadruplicar a CTC do substrato exposto $(\mathrm{r}=0,71)$ e prover ao substrato revegetado um valor de CTC $30 \%$ superior ao mensurado no solo sob Cerrado (Quadro 2). Entretanto, a matéria orgânica mostrou fraca correlação $(r=0,58)$ com a saturação por bases $(V \%)$. Lodos de esgotos não são fontes expressivas de bases (Corrêa et al., 2010), e a adição de $20 \mathrm{Mg} \mathrm{ha}^{-1}$ de $\mathrm{CaOH}$ ao substrato exposto não foi capaz de manter elevada a V \% do substrato após uma década dos trabalhos de revegetação (Quadro 2).

O efeito químico mais acentuado da incorporação de lodo de esgoto ao substrato exposto foi a elevação de P-disponível para teores 13 vezes superiores aos medidos no solo sob Cerrado (Quadro 2). O lodo de esgoto é uma boa fonte de $\mathrm{P}$ (Quadro 1), e a elevação das concentrações desse nutriente e de $\mathrm{N}$ e $\mathrm{Zn}$ em solos e substratos tratados com esse resíduo é comum (Bezerra et al., 2006; Corrêa et al., 2010). No entanto, após uma década da incorporação de lodo de esgoto, o $\mathrm{pH}$ do substrato revegetado correlacionou-se melhor com a disponibilidade de $\mathrm{P}(\mathrm{r}=0,86)$ do que com o seu teor de matéria orgânica $(r=0,74)$.

A incorporação de $250 \mathrm{Mg} \mathrm{ha}^{-1}$ de lodo de esgoto ao substrato exposto em 1997 resultou na adição de 13,7 Mg ha-1 de matéria orgânica (Quadro 1). Portanto, a dose de aplicação de lodo de esgoto não é 
capaz de explicar sozinha $29,7 \mathrm{~g} \mathrm{~kg}^{-1}$ de matéria orgânica existente no substrato revegetado uma década depois (2008). A incorporação do resíduo foi capaz de criar condições para o estabelecimento e a permanência de uma cobertura vegetal sobre o substrato minerado (Calgaro et al., 2008; Carneiro et al., 2008; Corrêa et al., 2010), e o estrato herbáceo contribuiu para a elevação do teor de matéria a níveis $30 \%$ superiores aos medidos no solo sob Cerrado. O material orgânico e a cobertura vegetal criaram, dessa forma, um ambiente edáfico, cujos atributos químicos apresentavam um valor de indicador (Iz) duas vezes superior $(\mathrm{Iz}=202 \%)$ à qualidade mensurada no solo sob Cerrado (Figura 2). A elevada concentração de Pdisponível no substrato revegetado em relação ao solo controle contribuiu sobremaneira para esse valor de Iz.

\section{Qualidade biológica}

A comunidade de organismos edáficos é influenciada pela quantidade e pela qualidade do material orgânico no solo (Doran \& Zeiss, 2000). Entretanto, a matéria orgânica no substrato revegetado não se relacionou significativamente com a abundância da macrofauna de solo e apresentou fraca correlação $(r=0,50)$ com a diversidade de morfoespécies $\left(\mathrm{SCI}_{\mathrm{M}}\right)$. Esses resultados, os hábitos e as estratégias de sobrevivência desse grupo (Wolters, 2000; Kaschuk et al., 2006) sugerem que a macrofauna identificada utilizava-se mais da vegetação na jazida do que da matéria orgânica no substrato.

A matéria orgânica é um atributo químico, mas tem sido utilizada como indicador único de qualidade biológica de solos (Pignataro Netto et al., 2009) ou como indicador de microrganismos de solo (Araújo, 2004; Araújo et al., 2007), que são importantes para a existência de organismos macroscópicos no solo (Doran \& Zeiss, 2000). Assim, a presença de macrofauna no substrato revegetado denota a existência de uma cadeia trófica do ambiente edáfico (Dajoz, 2005), cuja base é representada por microrganismos que dependem diretamente da matéria orgânica (Pignataro Netto et al., 2009).

A abundância de representantes da macrofauna no substrato revegetado é cerca de $30 \%$ superior à encontrada no solo sob Cerrado (Quadro 2). Todavia, após uma década da revegetação, a diversidade no substrato revegetado limitava-se a $74 \%$ da existente no solo nativo: $\mathrm{SCI}_{\mathrm{M}}=0,65$ para o solo sob Cerrado e 0,48 para o substrato revegetado. De acordo com Gray (1999), $\mathrm{SCI}_{\mathrm{M}}=0,65$ representa alta diversidade e 0,48 reflete uma média diversidade no ambiente edáfico. Brachiaria decumbens responde pela quase totalidade da cobertura vegetal da jazida; a baixa variação vegetal limita a diversidade de espécies de solo e aumenta a abundância dos organismos que conseguem estabelecer-se em um local (Nunes et al., 2009). A relação desproporcional entre abundância de indivíduos e diversidade de espécies é típica de ambientes em estádios iniciais de sucessão (Dajoz, 2005).
Entre os representantes da macrofauna de solo identificados, os isópteros (térmitas) foram os mais abundantes, seguidos pelos himenópteros (formigas) e coleópteros (besouros). As três primeiras posições de abundância dos grupos identificados mantiveram-se iguais no solo sob Cerrado e no substrato revegetado. Contudo, os coleópteros foram significativamente mais abundantes no substrato revegetado do que no solo sob Cerrado (Quadro 3). Essa ordem é colonizadora de ambientes perturbados ou em estádios iniciais de sucessão, especialmente aqueles com considerável disponibilidade de material orgânico (Wolters, 2000; Dajoz, 2005).

Grande habilidade para escavar e alta capacidade de transportar material orgânico para as camadas mais profundas são as características predominantes da macrofauna de solo (Vargas \& Hungria, 1997; Kaschuk et al., 2006). Térmitas, formigas, larvas de coleópteros e anelídeos são considerados "engenheiros do ecossistema", pois suas atividades levam à criação de estruturas biogênicas (galerias, ninhos, câmaras e bolotas fecais), que modificam as propriedades edáficas e disponibilizam recursos para outros organismos (Wolters, 2000). Essas atividades e estruturas são essenciais para a formação de solos, sobretudo em regiões tropicais (Vargas \& Hungria, 1997).

O valor do indicador de qualidade biológica para o substrato revegetado foi similar ao do solo sob Cerrado $(\mathrm{Iz}=102 \%)$. A menor diversidade de morfoespécies no substrato revegetado foi compensada pela maior abundância de indivíduos da macrofauna, que são essenciais para a atividade construtiva de solo no estádio de recuperação em que a área se encontra.

\section{Quadro 3. Número de indivíduos da fauna coletados no substrato revegetado e no solo sob Cerrado de acordo com a Classe ou Ordem (média \pm desvio-padrão, $n=10$ )}

\begin{tabular}{lrr}
\hline Classe/ordem & \multicolumn{2}{c}{ Número de indivíduos ${ }^{(\mathbf{1})}$} \\
\cline { 2 - 3 } & $\begin{array}{c}\text { Substrato } \\
\text { revegetado }\end{array}$ & $\begin{array}{c}\text { Solo sob } \\
\text { Cerrado }\end{array}$ \\
\hline Isópteros & $19,3 \mathrm{a}$ & $12,1 \mathrm{a}$ \\
Himenópteros & $5,7 \mathrm{a}$ & $8,1 \mathrm{a}$ \\
Coleópteros & $1,5 \mathrm{a}$ & $0,2 \mathrm{~b}$ \\
Anelídeos & $0,6 \mathrm{a}$ & $0,1 \mathrm{~b}$ \\
Ortópteros & $0,2 \mathrm{a}$ & $0,2 \mathrm{a}$ \\
Aracnídeos & $<0,1$ & 0,1 \\
Miriápodes & $<0,1$ & $<0,1$ \\
ni1 & $7,3 \mathrm{a}$ & $4,4 \mathrm{a}$ \\
ni2 & $7,9 \mathrm{a}$ & $1,9 \mathrm{~b}$ \\
ni3 & $<0,1 \mathrm{a}$ & $4,3 \mathrm{~b}$ \\
ni4 & $1,1 \mathrm{a}$ & $0,9 \mathrm{a}$ \\
ni5 & $<0,1 \mathrm{a}$ & $1,0 \mathrm{~b}$ \\
Total & $43,6 \mathrm{a}$ & $33,3 \mathrm{~b}$ \\
\hline
\end{tabular}

(1) n.i: não identificado. Médias $(\mathrm{n}=10)$ seguidas de mesma letra em cada linha não diferem pelo teste $t(p<0,05)$. 


\section{Qualidade do substrato revegetado}

A composição dos índices (Iz) de qualidade física, química e biológica em um único índice de qualidade do substrato revegetado (IQ) mostrou que ele extrapolou os valores do solo considerado ideal em $34 \%$ (Quadro 4). O valor do indicador de qualidade química $\left(\mathrm{I}_{\mathrm{Z}}=202 \%\right)$ causou essa extrapolação, e a concentração de P-disponível no substrato revegetado foi responsável pelo alto valor do Iz químico. Por outro lado, os atributos físicos mensurados no substrato revegetado permaneceram significativamente aquém dos mensurados no solo sob Cerrado. Caso o objetivo da recuperação seja a restauração do ecossistema no local, técnicas que melhorem a qualidade física do substrato e não excedam os níveis de fertilidade química devem ser desenvolvidas.

\section{Quadro 4. Índice de qualidade (IQ)}

\begin{tabular}{lcc}
\hline \multicolumn{1}{c}{ Material } & $\begin{array}{c}\text { Área geométrica do } \\
\text { triângulo (adimensional) }\end{array}$ & IQ \\
\hline & & $\%$ \\
Substrato revegetado & $13,4 \times 10^{3}$ & 134 \\
Solo sob Cerrado & $10,0 \times 10^{3}$ & 100 \\
\hline
\end{tabular}

\section{CONCLUSÕES}

1. A incorporação de lodo de esgoto ao substrato minerado reabilitou funções edáficas que permitiram o estabelecimento de uma cobertura vegetal sobre a jazida minerada.

2. A incorporação de lodo de esgoto ao substrato minerado e o desenvolvimento de uma cobertura vegetal herbácea resultaram em melhorias químicas e biológicas do substrato exposto.

3. A dose de lodo de esgoto utilizada (250 $\mathrm{Mg} \mathrm{ha}^{-1}$ ) resultou na incorporação excessiva de $\mathrm{P}$ ao substrato.

4. Os atributos físicos do substrato revegetado são, entre os analisados, os menos semelhantes aos do solo original sob Cerrado.

\section{LITERATURA CITADA}

ADANI, F.; GENEVINI, P.; RICCA, R.; TAMBONE, F. \& MONTONERI, E. Modification of soil humic matter after 4 years of compost application. Waste Manag., 27:319. 324, 2007.

ALVES, M.C.; SUZUKI. L.G.A.S. \& SUZUKI, L.E.A.S. Densidade do solo e infiltração de água como indicadores da qualidade física de um Latossolo Vermelho distrófico em recuperação. R. Bras. Ci. Solo, 31:617-625, 2007.
ARAUJO, G.H.M.F. Efeito do manejo sobre a qualidade do substrato e o desenvolvimento de espécies arbóreas do Cerrado em uma cascalheira no Distrito Federal. Brasília, Universidade de Brasília, 2006. 33p. (Tese de Mestrado)

ARAÚJO, R. Avaliação da qualidade do solo em áreas sob diferentes usos. Brasília, Universidade de Brasília, 2004. 77p. (Tese de Mestrado)

ARAÚJO, R.; GOEDERT, W.J. \& LACERDA, M.P.C. Qualidade do solo sob diferentes usos e sob Cerrado nativo. R. Bras. Ci. Solo, 31:1099-1108, 2007.

BEZERRA, F.B.; OLIVEIRA, M.A.C.L.; PEREZ, D.V.; ANDRADE, A.G. \& MENEGUELLI, N.A. Lodo de esgoto em revegetação de área degradada. Pesq. Agropec. Bras., 41:469-476, 2006.

BRADY, N.C. \& WEIL, R.R. The nature and properties of soils. 13.ed. New Jersey, Prentice-Hall, 2002. 960p.

CALGARO, H.F.; VALÉRIO FILHO, W.V.; MALTONI, K.L.; AQUINO, A.M.S.S. \& CASSIOLATO, R. Adubação química e orgânica na recuperação da fertilidade de subsolo degradado e na micorrização do Stryphnodendron polyphyllum. R. Bras. Ci. Solo, 32:1337-1347, 2008.

CAMPOS, M.L.; ALMEIDA, J.A. \& SOUZA, L.S. Avaliação de três áreas de solo construído após mineração de carvão a céu aberto em Lauro Muller, Santa Catarina. R. Bras. Ci. Solo, 27:1123-1137, 2003.

CARNEIRO, M.A.C.; SIQUEIRA, J.O.; MOREIRA, F.M.S. \& SOARES, A.L.L. Carbono orgânico, $\mathrm{N}$ total, biomassa e atividade microbiana do solo em duas cronossequências de reabilitação após a mineração de bauxita. R. Bras. Ci. Solo, 32:621-632, 2008.

CORRÊA, R.S.; BIAS, E.S. \& BAPTISTA, G.M.M. Áreas degradadas pela mineração no Distrito Federal. In: CORRÊA, R.S. \& BAPTISTA, G.M.M., orgs. Mineração e áreas degradadas no Cerrado. Brasília, Universa, 2004. p.9-21.

CORRÊA, R.S.; SILVA, L.C.R.; BAPTISTA, G.M.M. \& SANTOS, P.F. Fertilidade química de um substrato tratado com lodo de esgoto e composto de resíduos domésticos. R. Bras. Eng. Agríc. Amb., 14:538-544, 2010.

DAJOZ, R. Princípios de ecologia. 7.ed. Porto Alegre, Artmed, 2005. 519p.

DORAN, J.W. \& ZEISS, M.R. Soil health and sustainability: Managing the biotic component of soil quality. Appl. Soil Ecol., 15:3-11, 2000.

EMPRESA BRASILEIRA DE PESQUISA AGROPECUÁRIA EMBRAPA. Serviço Nacional de Levantamento e Conservação de Solos. Manual de métodos de análise de solos. 2.ed. Rio de Janeiro, 1997. 212p.

EMPRESA BRASILEIRA DE PESQUISA AGROPECUÁRIA EMBRAPA. Centro Nacional de Pesquisa de Solos. Sistema brasileiro de classificação de solos. Rio de Janeiro, 1999. 412p.

GOEDERT, W.J. Qualidade do solo em sistemas de produção agrícola. In: CONGRESSO BRASILEIRO DE CIÊNCIA DO SOLO, 30., Recife, 2005. Anais... Recife, Sociedade Brasileira de Ciência do Solo, 2005. p.1-20. 
GOEDERT, W.J. \& CORRÊA, R.S. Usos, degradação e qualidade do solo. In: CORRÊA, R.S. \& BAPTISTA, G.M.M., orgs. Mineração e áreas degradadas no Cerrado. Brasília, Universa, 2004. p.159-172.

GRAY, N.F. Water technology: An introduction for environmental scientists and engineers. London, Arnold, 1999. 538p.

KASCHUK, G.; SANTOS, J.C.P.; ALMEIDA, J.A.; SINHORATI, D.C. \& BERTON-JUNIOR, J.F. Termite activity in relation to natural grassland soil attributes. Sci. Agric., 63:583$588,2006$.

KIEHL, E.J. Manual de edafologia. São Paulo, Ceres, 1979. $262 p$.

LEITE, L.L.; MARTINS, C.R. \& HARIDASAN, M. Efeitos da descompactação e adubação do solo na revegetação espontânea de uma cascalheira no Parque Nacional de Brasília. In: SIMPÓSIO SUL-AMERICANO, 1.; SIMPÓSIO NACIONAL SOBRE RECUPERAÇÃO DE ÁREAS DEGRADADAS, 2., Curitiba, 1994. Anais... Curitiba, Sobrade, 1994. p.527-534

MALAVOLTA, E. Manual de calagem e adubação das principais culturas. São Paulo, Ceres, 1987. 496p.

NUNES, L.A.P.L; FILHO, J.A.A. \& MENEZES, R.I.Q. Diversidade da fauna edáfica em solos submetidos a diferentes sistemas de manejo no semi-árido nordestino. Sci. Agr., 10:43-49, 2009.

OLIVEIRA, G.C.; SEVERIANO, E.C. \& MELLO, C.R. Dinâmica da resistência à penetração de um Latossolo Vermelho da Microrregião de Goiânia. R. Bras. Eng. Agríc. Amb., $1: 265-270,2007$.
PIGNARO NETTO, I.T.; KATO, E. \& GOEDERT, W.J. Atributos físicos e químicos de um Latossolo VermelhoAmarelo sob pastagens com diferentes históricos de uso. R. Bras. Ci. Solo, 33:1441-1448, 2009.

REIS, L.L. Monitoramento da recuperação ambiental de áreas de mineração de bauxita na Floresta Nacional de SaracáTaquera, Porto Trombetas (PA). Rio de Janeiro, Universidade Federal Rural do Rio de Janeiro, 2006. 175p. (Tese de Doutorado)

SIMONETE, M.A.; KIEHL, J.C.; ANDRADE, C.A. \& TEIXEIRA, C.F.A. Efeito do lodo de esgoto em um Argissolo e no crescimento e nutrição de milho. Pesq. Agropec. Bras., 38:1187-1195, 2003.

STOLF, R.; FERNANDES, J. \& FURLANI, V.L.N. Penetrômetro de impacto modelo IAA/PLANALSUCARStolf: Recomendação para seu uso. Piracicaba, STAB, 1983. p.18-23.

STOLF, R. Teoria e teste experimental de fórmulas de transformação dos dados de penetrômetros de impacto em resistência do solo. R. Bras. Ci. Solo, 15:229-235, 1991.

VALCARCEL, R.; VALENTE, F.D.W.; MOROKAWA, M.J.; CUNHA NETO, F.V. \& PEREIRA, C.R. Avaliação da biomassa de raízes finas em área de empréstimo submetida a diferentes composições de espécies. R. Árvore, 31:923-930, 2007.

VARGAS, M.A.T. \& HUNGRIA, M. Biologia dos solos dos Cerrados. Planaltina, Embrapa Cerrados, 1997. 524p.

WOLTERS, V. Invertebrate control of soil organic matter stability. Biol. Fert. Soils, 31:1-19, 2000. 